\title{
INVERSE PROBLEM FOR THE PARABOLIC EQUATION WITH GENERAL WEAK DEGENERATION
}

\author{
PhD (Candidate of Physics and Mathematics) ${ }^{1}$ Huzyk Nadiia, \\ $P h D$ (Candidate of Physics and Mathematics) ${ }^{2}$ Brodyak Oksana, \\ PhD (Candidate of Technical Sciences) ${ }^{1}$ Lishchynska Khrystyna, \\ ${ }^{I}$ Department of Engineering Mechanics (Weapons and Equipment of Military Engineering Forces), \\ Hetman Petro Sahaidachnyi National Army Academy, Lviv, Ukraine \\ ${ }^{2}$ National University "Lviv Polytechnic", Lviv, Ukraine
}

DOI: https://doi.org/10.31435/rsglobal_conf/30052021/7574

\begin{abstract}
In the rectangle with known boundary we consider a coefficient inverse problem for the degenerate parabolic equation with given initial condition, Dirichlet boundary conditions and the values of the heat moments as the overdetermination conditions. The minor coefficient of the equation is a polynomial of the first power with respect to the space variable with two unknown time-dependent coefficients. The degeneration of the equation is caused by the power function at the time derivative. Applying the Schauder fixed-point theorem we establish conditions of existence of the classical local in time solution to this problem. The global uniqueness is based on the properties of the solutions of the integral Voltera equations of second kind with integrable kernels. The case of weak degeneration is investigated.
\end{abstract}

Keywords: inverse problem, minor coefficient, parabolic equation, weak power degeneration, integral overdetermination condition.

Introduction. Inverse problems arise when under given consequences we have to find the reasons causing them. Investigation of such problems is urged by the need of solving problems applying in geophysics, medicine, engineering, finance and other sciences. In this paper, we will talk about inverse problems with unknown time-dependent coefficients. Degenerate parabolic problems arise in a lot of fields of nature and sciences. Applications of such problems include the mathematical model of the flow in a porous media, propagation of the thermal waves in plasma and others.

For today the inverse problems of determination of the time-dependent both major and minor coefficients in the parabolic equation without degeneration are studied sufficiently full (see [1]-[6] and a bibliography of them).

In the papers [7]-[9] the conditions of the correct solvability of the inverse problems of identification of the time-dependent major coefficient in the degenerate parabolic equation are established. Both cases of weak $(0<\beta<1)$ and strong $(\beta \geq 1)$ power degeneration are investigated.

The conditions for existence and uniqueness of classical solutions of inverse problems of identification of the minor coefficient in the parabolic equation

$$
u_{t}=a(t) t^{\beta} u_{x x}+b(t) u_{x}+c(x, t) u+f(x, t)
$$

for different sets of boundary and overdetermination conditions are found in [10], [11], while the simultaneous determination of two coefficients $a=a(t), b=b(t)-$ in [12]. In the paper [13] it is investigated the inverse problem for determination of the coefficient $a=a(t), a(t)>0, t \in[0, T]$ in the parabolic equation with degeneration at the time derivative of unknown function.

The goal of this paper is to establish the conditions of existence and uniqueness of the classical solution to the inverse problem for determination of the time-dependent functions in the minor coefficient in a parabolic equation with degeneration caused by the power function at the time derivative of unknown function.

1. Statement of the problem. In a domain $Q_{T}=\{(x, t): 0<x<h, 0<t<T\}$ we consider an inverse problem for determination of the time-dependent coefficients $b_{1}=b_{1}(t), b_{2}=b_{2}(t)$, in the onedimensional parabolic equation

$$
t^{\beta} u_{t}=a(t) u_{x x}+\left(b_{1}(t) x+b_{2}(t)\right) u_{x}+c(x, t) u+f(x, t)
$$


with initial condition

$$
u(x, 0)=\varphi(x), \quad x \in[0, h]
$$

boundary conditions

$$
u(0, t)=\mu_{1}(t), \quad u(h, t)=\mu_{2}(t), \quad t \in[0, T]
$$

and overdetermination condition

$$
\begin{aligned}
& \int_{0}^{h} u(x, t) d x=\mu_{3}(t), \quad t \in[0, T], \\
& \int_{0}^{h} x u(x, t) d x=\mu_{4}(t), \quad t \in[0, T] .
\end{aligned}
$$

Definition. A triplet of functions $\left(b_{1}, b_{2}, u\right) \in(C[0, T])^{2} \times C^{2,1}\left(Q_{T}\right) \cap C^{1,0}\left(\overline{Q_{T}}\right)$ is called a solution to the problem (1)-(5) if it verifies the equation (1) and conditions (2)-(5).

We will investigate the case of weak degeneration, when $0<\beta<1$. Applying the Schauder fixed-point theorem there is established conditions of existence of the solution to the named problem. The proof of the uniqueness is based on the properties of the solutions to the homogeneous integral Voltera equations of the second kind with integrable kernels.

\section{Existence of solution to the problem (1)-(5).}

Theorem 1. Suppose that the following conditions hold:

A1) $\varphi \in C^{2}[0, h], \mu_{i} \in C^{1}[0, T], i=\overline{1,4}, a \in C[0, T], a(t)>0, t \in[0, T], c, f \in C\left(\overline{Q_{T}}\right)$ and satisfy the Hölder condition with respect to $x$ uniformly to $t$ with indicator $\alpha, 0<\alpha<1$;

A2) $\left(h \mu_{2}(t)-\mu_{3}(t)\right)^{2}-\left(\mu_{2}(t)-\mu_{1}(t)\right)\left(h^{2} \mu_{2}(t)-2 \mu_{4}(t)\right) \neq 0, \quad t \in[0, T]$;

A3) $\mu_{1}(0)=\varphi(0), \mu_{2}(0)=\varphi(h), \int_{0}^{h} \varphi(x) d x=\mu_{3}(0), \int_{0}^{h} x \varphi(x) d x=\mu_{4}(0)$.

Then the problem (1)-(5) has the solution $\left(b_{1}, b_{2}, u\right)$ for $x \in[0, h]$ and $t \in\left[0, T_{0}\right]$, where the number $T_{0}, 0<T_{0}<T$ is defined by the problem data.

Proof. First, we reduce the problem (1)-(5) to the equivalent system of equations. For this aim, we make the substitution in the problem (1)-(5):

$$
u(x, t)=\widetilde{u}(x, t)+\varphi(x)-\varphi(0)+\mu_{1}(t)+\frac{x}{h}\left(\mu_{2}(t)-\mu_{1}(t)-\mu_{2}(0)+\mu_{1}(0)\right) .
$$

As a result we obtain the equation with respect to the function $\tilde{u}(x, t)$ :

$$
\begin{aligned}
& t^{\beta} \widetilde{u}_{t}=a(t) \widetilde{u}_{x x}+\left(b_{1}(t) x+b_{2}(t)\right) \tilde{u}_{x}+c(x, t) \widetilde{u}+f(x, t)-t^{\beta} \mu_{1}^{\prime}(t)-\frac{t^{\beta} x}{h}\left(\mu_{2}^{\prime}(t)-\mu_{1}^{\prime}(t)\right)+ \\
& +a(t) \varphi^{\prime \prime}(x)+\left(b_{1}(t) x+b_{2}(t)\right)\left(\varphi^{\prime}(x)+\frac{1}{h}\left(\mu_{2}(t)-\mu_{1}(t)-\mu_{2}(0)+\mu_{1}(0)\right)\right)+ \\
& +c(x, t)\left(\varphi(x)-\varphi(0)+\mu_{1}(t)+\frac{x}{h}\left(\mu_{2}(t)-\mu_{1}(t)-\mu_{2}(0)+\mu_{1}(0)\right)\right)
\end{aligned}
$$

and homogeneous initial and boundary conditions

$$
\begin{gathered}
\tilde{u}(x, 0)=0, x \in[0, h], \\
\tilde{u}(0, t)=\tilde{u}(h, t)=0, t \in[0, T] .
\end{gathered}
$$

The problem (7)-(9) is equivalent to the integro-differential equation

$$
\begin{gathered}
\tilde{u}(x, t)=\int_{00}^{t h} G_{1}(x, t, \xi, \tau)\left(\left(b_{1}(\tau) \xi+b_{2}(\tau)\right) \tilde{u}_{\xi}(\xi, \tau)+f(\xi, \tau)-\tau^{\beta} \mu_{1}{ }^{\prime}(\tau)-\frac{\tau^{\beta} \xi}{h}\left(\mu_{2}{ }^{\prime}(\tau)-\mu_{1}{ }^{\prime}(\tau)\right)+\right. \\
\quad+a(\tau) \varphi^{\prime \prime}(\xi)+\left(b_{1}(\tau) \xi+b_{2}(\tau)\right)\left(\varphi^{\prime}(\xi)+\frac{1}{h}\left(\mu_{2}(\tau)-\mu_{1}(\tau)-\mu_{2}(0)+\mu_{1}(0)\right)\right)+ \\
\left.+c(\xi, \tau)\left(\varphi(\xi)-\varphi(0)+\mu_{1}(\tau)+\frac{\xi}{h}\left(\mu_{2}(\tau)-\mu_{1}(\tau)-\mu_{2}(0)+\mu_{1}(0)\right)\right)\right) d \xi d \tau, \quad(x, t) \in \overline{Q_{T}} .
\end{gathered}
$$


We denote by $G_{1}(x, t, \xi, \tau)$ the Green function of the first boundary-value problem for the equation

$$
t^{\beta} u_{t}=a(t) u_{x x} .
$$

Put $v(x, t) \equiv u_{x}(x, t)$. Using (6), (10), we reduce the direct problem (1)-(3) by the system of integral equations with respect to unknown functions $u=u(x, t), v=v(x, t)$ :

$$
\begin{gathered}
u(x, t)=\varphi(x)-\varphi(0)+\mu_{1}(t)+\frac{x}{h}\left(\mu_{2}(t)-\mu_{1}(t)-\mu_{2}(0)+\mu_{1}(0)\right)+ \\
+\int_{00}^{t h} G_{1}(x, t, \xi, \tau)\left(\left(b_{1}(\tau) \xi+b_{2}(\tau)\right) v(\xi, \tau)+f(\xi, \tau)-\tau^{\beta} \mu_{1}{ }^{\prime}(\tau)-\frac{\tau^{\beta} \xi}{h}\left(\mu_{2}{ }^{\prime}(\tau)-\mu_{1}{ }^{\prime}(\tau)\right)+\right. \\
\left.+a(\tau) \varphi^{\prime \prime}(\xi)+c(\xi, \tau)\left(\varphi(\xi)-\varphi(0)+\mu_{1}(\tau)+\frac{\xi}{h}\left(\mu_{2}(\tau)-\mu_{1}(\tau)-\mu_{2}(0)+\mu_{1}(0)\right)\right)\right) d \xi d \tau, \\
v(x, t)=\varphi^{\prime}(x)+\frac{1}{h}\left(\mu_{2}(t)-\mu_{1}(t)-\mu_{2}(0)+\mu_{1}(0)\right)+ \\
+\int_{00}^{t h} G_{1 x}(x, t, \xi, \tau)\left(\left(b_{1}(\tau) \xi+b_{2}(\tau)\right) v(\xi, \tau)+f(\xi, \tau)-\tau^{\beta} \mu_{1}{ }^{\prime}(\tau)-\frac{\tau^{\beta} \xi}{h}\left(\mu_{2}{ }^{\prime}(\tau)-\mu_{1}{ }^{\prime}(\tau)\right)+\right. \\
\left.+a(\tau) \varphi^{\prime \prime}(\xi)+c(\xi, \tau)\left(\varphi(\xi)-\varphi(0)+\mu_{1}(\tau)+\frac{\xi}{h}\left(\mu_{2}(\tau)-\mu_{1}(\tau)-\mu_{2}(0)+\mu_{1}(0)\right)\right)\right) d \xi d \tau,
\end{gathered}
$$

We obtain the equation (11) differentiating (10) with respect to space variable $x$. To find an equation for the functions $b_{1}=b_{1}(t), b_{2}=b_{2}(t)$ we multiply the equation (1) by $x^{k}, k=0,1$ respectively and integrate it using the conditions (2)-(5):

$$
\begin{gathered}
b_{1}(t)=\Delta^{-1}\left(\left(t^{\beta} \mu_{3}{ }^{\prime}(t)-a(t)(v(h, t)-v(0, t))-\int_{0}^{h}(c(x, t) u(x, t)+f(x, t)) d x\right)\left(h \mu_{2}(t)-\mu_{3}(t)\right)-\right. \\
\left.-\left(t^{\beta} \mu_{4}{ }^{\prime}(t)-a(t)\left(h v(h, t)-\mu_{2}(t)+\mu_{1}(t)\right)-\int_{0}^{h} x(c(x, t) u(x, t)+f(x, t)) d x\right)\left(\mu_{2}(t)-\mu_{1}(t)\right)\right), \quad t \in[0, T], \\
b_{2}(t)=\Delta^{-1}\left(\left(t^{\beta} \mu_{4}{ }^{\prime}(t)-a(t)\left(h v(h, t)-\mu_{2}(t)+\mu_{1}(t)\right)-\int_{0}^{h} x(c(x, t) u(x, t)+f(x, t)) d x\right)\left(h \mu_{2}(t)-\mu_{3}(t)\right)-\right. \\
-\left(t^{\beta} \mu_{3}{ }^{\prime}(t)-a(t)(v(h, t)-v(0, t))-\int_{0}^{h}(c(x, t) u(x, t)+f(x, t)) d x\right)\left(h^{2} \mu_{2}(t)-2 \mu_{4}(t)\right), \quad t \in[0, T] .
\end{gathered}
$$

Thus the inverse problem (1)-(5) we reduce to the equivalent system of equations (11)-(14). It means that if a triplet of functions $\left(b_{1}, b_{2}, u\right)$ is a solution to the problem (1)-(5), then $\left(u, v, b_{1}, b_{2}\right)$ is a continuous solution to the system of equations (11)-(14) and contrary if $\left(u, v, b_{1}, b_{2}\right) \in\left(C\left(\overline{Q_{T}}\right)\right)^{2} \times(C[0, T])^{2}$ is a solution to the system of equations (11)-(14) then $\left(b_{1}, b_{2}, u\right)$ is a solution to the problem (1)-(5).

Now we prove the existence of the solution to the system of equation (11)-(14). First of all we establish the behavior of the integrals in the right-hand sides of the formulas (11), (12). Taking into account the known estimate [14, p. 469]

$$
\left|D_{t}^{r} D_{y}^{s} G(y, t, \eta, \tau)\right| \leq C_{1}(\theta(t)-\theta(\tau))^{-\frac{1+2 r+s}{2}} \exp \left(-C_{2} \frac{(y-\eta)^{2}}{\theta(t)-\theta(\tau)}\right)
$$

where $r \in\{0,1\}, \quad s \in\{0,1,2\}, 2 r+s=1$ or $2 r+s=2, \tau<t$ and $\theta(t)=\int_{0}^{t} \sigma^{-\beta} d \sigma$, we obtain 


$$
\begin{aligned}
& J_{1} \equiv \mid \int_{00}^{t h} G_{1}(x, t, \xi, \tau)\left(\left(b_{1}(\tau) \xi+b_{2}(\tau)\right) v(\xi, \tau)+f(\xi, \tau)-\tau^{\beta} \mu_{1}{ }^{\prime}(\tau)-\frac{\tau^{\beta} \xi}{h}\left(\mu_{2}{ }^{\prime}(\tau)-\mu_{1}{ }^{\prime}(\tau)\right)+\right. \\
& \left.+a(\tau) \varphi^{\prime \prime}(\xi)+c(\xi, \tau)\left(\varphi(\xi)-\varphi(0)+\mu_{1}(\tau)+\frac{\xi}{h}\left(\mu_{2}(\tau)-\mu_{1}(\tau)-\mu_{2}(0)+\mu_{1}(0)\right)\right)\right) d \xi d \tau \leq \\
& \leq C_{3} \int_{0}^{t} \int_{0}^{h}(\theta(t)-\theta(\tau))^{-\frac{1}{2}} \exp \left(-C_{2} \frac{(x-\xi)^{2}}{\theta(t)-\theta(\tau)}\right) d \xi d \tau \leq C_{4} t
\end{aligned}
$$

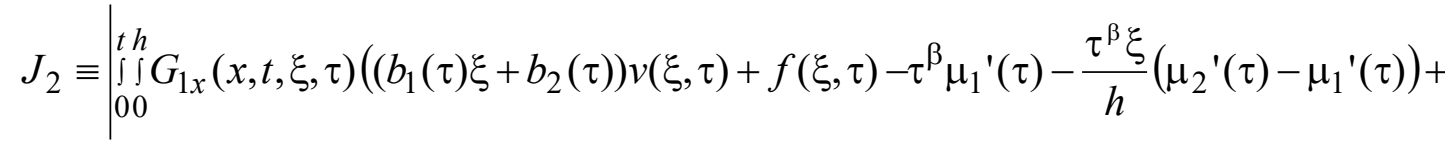

$$
\begin{aligned}
& \left.+a(\tau) \varphi^{\prime \prime}(\xi)+c(\xi, \tau)\left(\varphi(\xi)-\varphi(0)+\mu_{1}(\tau)+\frac{\xi}{h}\left(\mu_{2}(\tau)-\mu_{1}(\tau)-\mu_{2}(0)+\mu_{1}(0)\right)\right)\right) d \xi d \tau \leq \\
& \leq C_{5} \int_{0}^{t h}(\theta(t)-\theta(\tau))^{-1} \exp \left(-C_{2} \frac{(x-\xi)^{2}}{\theta(t)-\theta(\tau)}\right) d \xi d \tau \leq C_{6} \int_{0}^{t} \frac{d \tau}{\sqrt{\theta(t)-\theta(\tau)}} \leq C_{7} t^{\frac{1+\beta}{2}} \int_{0}^{1} \frac{d z}{\sqrt{1-z^{1+\beta}}} .
\end{aligned}
$$

It means that integrals in the right-hand sides of the formulas (11), (12) tend to zero when $t \rightarrow 0$.

We represent the system of equations (11)-(14) as an operator equation

$$
W=P W
$$

where $W=\left(u, v, b_{1}, b_{2}\right)$ and the operator $P$ is determined by the right-hand sides of the equations (10)-(14) respectively.

Let $|u(x, t)| \leq M_{1},|v(x, t)| \leq M_{2}, \quad(x, t) \in \bar{Q}_{T}, \quad$ where $M_{1}, M_{2} \quad$ denote some positive constants. We will choose them below. Then from (13), (14) we deduce

$$
\begin{gathered}
\left|P_{3} W\right| \leq \frac{C_{8}\left(1+M_{1}+M_{2}\right)}{\min |\Delta|} \equiv M_{3}, t \in[0, T], \\
\left|P_{4} W\right| \leq \frac{C_{9}\left(1+M_{1}+M_{2}\right)}{\min |\Delta|} \equiv M_{4}, t \in[0, T] .
\end{gathered}
$$

Using this estimates in (11), (12) we find

$$
\begin{gathered}
\left|P_{1} W\right| \leq C_{10} t+\max _{(x, t) \in \bar{Q}_{T} \mid} \mid \varphi(x)-\varphi(0)+\mu_{1}(t)+\frac{x}{h}\left(\mu_{2}(t)-\mu_{1}(t)-\mu_{2}(0)+\mu_{1}(0)\right), t \in[0, T], \\
\left|P_{2} W\right| \leq C_{11} t^{\frac{1+\beta}{2}}+\max _{(x, t) \in \bar{Q}_{T}} \mid \varphi^{\prime}(x)+\frac{1}{h}\left(\mu_{2}(t)-\mu_{1}(t)-\mu_{2}(0)+\mu_{1}(0)\right), t \in[0, T] .
\end{gathered}
$$

Now we choose the number $M_{1}>\max _{(x, t) \in \bar{Q}_{T}}\left|\varphi(x)-\varphi(0)+\mu_{1}(t)+\frac{x}{h}\left(\mu_{2}(t)-\mu_{1}(t)-\mu_{2}(0)+\mu_{1}(0)\right)\right|$, $M_{2}>\max _{(x, t) \in \bar{Q}_{T}}\left|\varphi^{\prime}(x)+\frac{1}{h}\left(\mu_{2}(t)-\mu_{1}(t)-\mu_{2}(0)+\mu_{1}(0)\right)\right|$ and the number $T_{0}, 0<T_{0} \leq T$ such that

$$
\begin{gathered}
C_{10} T_{0}+\max _{(x, t) \in \bar{Q}_{T}}\left|\varphi(x)-\varphi(0)+\mu_{1}(t)+\frac{x}{h}\left(\mu_{2}(t)-\mu_{1}(t)-\mu_{2}(0)+\mu_{1}(0)\right)\right| \leq M_{1}, \\
C_{12} t^{\frac{1+\beta}{2}}+\max _{(x, t) \in \bar{Q}_{T}}\left|\varphi^{\prime}(x)+\frac{1}{h}\left(\mu_{2}(t)-\mu_{1}(t)-\mu_{2}(0)+\mu_{1}(0)\right)\right| \leq M_{2} .
\end{gathered}
$$


In the Banach space $B=\left(C\left(\bar{Q}_{T_{0}}\right)\right)^{2} \times\left(C\left[0, T_{0}\right]\right)^{2}$ we choose the set

$$
N=\left\{\left(u, v, b_{1}, b_{2}\right) \in \mathrm{B}:|u(x, t)| \leq M_{1},|v(x, t)| \leq M_{2},\left|b_{1}(t)\right| \leq M_{3},\left|b_{2}(t)\right| \leq M_{4}\right\} .
$$

We conclude due to obtaining a priori estimates that $N$ is convex and the operator $P$ maps it into itself. The compactness of the operator $P$ can be proved as in [13]. Thus, all conditions of the Schauder fixed-point theorem are fulfilled. It means that there exists the solution to the system of equations (10)-(14) when $(x, t) \in[0, h] \times\left[0, T_{0}\right]$ and correspondingly to the problem (1)-(5) when $(x, t) \in[0, h] \times\left[0, T_{0}\right]$. The Theorem 1 is proved.

\section{Uniqueness of solution to the problem (1)-(5).}

\section{Theorem 2. If the condition}

$$
\left(h \mu_{2}(t)-\mu_{3}(t)\right)^{2}-\left(\mu_{2}(t)-\mu_{1}(t)\right)\left(h^{2} \mu_{2}(t)-2 \mu_{4}(t)\right) \neq 0, \quad t \in[0, T]
$$

hold then the solution $\left(b_{1}, b_{2}, u\right)$ to the problem (1)-(5) is unique.

Proof. Suppose that the problem (1)-(5) has two solutions $\left(b_{1 i}, b_{2 i}, u_{i}\right), i=1,2$. The differences of these solutions we denote by $b_{1}(t)=b_{11}(t)-b_{12}(t), b_{2}(t)=b_{21}(t)-b_{22}(t), u(x, t)=u_{1}(x, t)-u_{2}(x, t)$. They satisfy the equation

$$
t^{\beta} u_{t}=a(t) u_{x x}+\left(b_{11}(t) x+b_{21}(t)\right) u_{x}+c(x, t) u+\left(b_{1}(t) x+b_{2}(t)\right) u_{2 x}, \quad(x, t) \in Q_{T}
$$

with conditions

$$
\begin{gathered}
u(x, 0)=0, \quad x \in[0, h], \\
u(0, t)=u(h, t)=0, \quad t \in[0, T], \\
\int_{0}^{h} u(x, t) d x=0, \quad t \in[0, T], \\
{ }_{0}^{h} x u(x, t) d x=0, \quad t \in[0, T] .
\end{gathered}
$$

With the aid of the Green function $G_{1}^{*}(x, t, \xi, \tau)$ of the first boundary-value problem for the equation

$$
t^{\beta} u_{t}=a(t) u_{x x}+\left(b_{11}(t) x+b_{21}(t)\right) u_{x}+c(x, t) u
$$

we represent the solution to the problem (20)-(22) in the form

$$
u(x, t)=\int_{00}^{t h} G_{1}^{*}(x, t, \xi, \tau)\left(b_{1}(\tau) \xi+b_{2}(\tau)\right) u_{2 \xi}(\xi, \tau) d \xi d \tau .
$$

Differentiating (32) with respect to $x$ we obtain

$$
u_{x}(x, t)=\int_{00}^{t h} \int_{1 x}^{*}(x, t, \xi, \tau)\left(b_{1}(\tau) \xi+b_{2}(\tau)\right) u_{2 \xi}(\xi, \tau) d \xi d \tau .
$$

Now we multiply the equation (20) by $x^{k}, k=0,1$ respectively and integrate it using the conditions (21)-(24):

$$
\begin{gathered}
b_{1}(t)=\Delta^{-1}\left(\left(a(t) h u_{x}(h, t)+\int_{0}^{h} x c(x, t) u(x, t) d x\right)\left(\mu_{2}(t)-\mu_{1}(t)\right)-\right. \\
\left.-\left(a(t)\left(u_{x}(h, t)-u_{x}(0, t)\right)+\int_{0}^{h} c(x, t) u(x, t) d x\right)\left(h \mu_{2}(t)-\mu_{3}(t)\right)\right), t \in[0, T] \\
b_{2}(t)=\Delta^{-1}\left(\left(a(t) h u_{x}(h, t)+\int_{0}^{h} x c(x, t) u(x, t) d x\right)\left(\mu_{2}(t)-\mu_{1}(t)\right)-\right. \\
\left.-\left(a(t)\left(u_{x}(h, t)-u_{x}(0, t)\right)+\int_{0}^{h} c(x, t) u(x, t) d x\right)\left(h^{2} \mu_{2}(t)-2 \mu_{4}(t)\right)\right), \quad t \in[0, T] .
\end{gathered}
$$


Substituting (26), (27) into (28), (29) we obtain the system of homogeneous integral Voltera equations of second kind with respect to $b_{1}=b_{1}(t), b_{2}=b_{2}(t)$. The kernels of this system are integrable since we investigate the case of weak degeneration. It implies that it has only trivial solution

$$
b_{1}(t)=0, b_{2}(t)=0, t \in[0, T] \text {. }
$$

Using this equality in the problem (20)-(22) we find

$$
u(x, t)=0,(x, t) \in[0, h] \times[0, T] .
$$

Theorem 2 is proved.

Remark. The degenerate parabolic equation can be generalized by changing the power function $t^{\beta}$ on monotone increasing function $\psi=\psi(t)$ such that $\psi(t)>0, t \in(0, T]$ and $\psi(0)=0$. It is evident that in the case of weak degeneration when $\lim _{t \rightarrow 0} \int_{0}^{t} \frac{d \tau}{\psi(\tau)}=0$ the statements of the Theorem 1 and Theorem 2 hold true.

Conclusion. There are established the sufficient conditions of existence and uniqueness of the classical solution to the inverse problem of identification of the time-dependent functions in the minor coefficient in the weakly degenerate parabolic equation.

\section{REFERENCES}

1. B. F. Jones, The determination of a coefficient in a parabolic differential equation. Part I. Existence and uniqueness, J. Math. Mech. 11, (1962), 907-918.

2. J. R. Cannon and W. Rundell, Recovering a time-dependent coefficient in a parabolic differential equation, J. Math. Anal. Appl. 160 (1991), 572-582.

3. Ivanchov M., Some inverse problems for the heat equation with non-local boundary conditions, Ukrain. Math. J. 45 (8), (1993), 1066-1071. (in Ukrainian)

4. Cannon J. R., Peres-Esteva S., Determination of the coefficient of $\mathrm{u}_{\mathrm{x}}$ in a linear parabolic equation, Inverse Problems 10 (3), (1993), 521-531.

5. Trong D. D., Ang D. D., Coefficient identification for a parabolic equation, Inverse Problems 10 (3), (1994), 733-752.

6. Pabyrivska N., Varenyk O., Identification of the minor coefficient in the parabolic equation, Visnyk Lviv. Univ. Ser. Mech.-Math. 64, (2005), 181-189. (in Ukrainian)

7. Ivanchov M., Saldina N., An inverse problem for a strongly degenerate heat equation, J. Inv. Ill-Posed Problem 14 (5), (2006), 1-16.

8. Ivanchov M., Saldina N., Inverse problem for the degenerate heat equation, Ukrain. Math. J. 57 (11), (2005), 1563-1570. (in Ukrainian)

9. Saldina N., Inverse problem for the parabolic equation with degeneration, Visnyk Lviv. Univ. Ser. Mech.Math. 64, (2005), 245-257. (in Ukrainian)

10. Hryntsiv N., Determination of the coefficient of the first derivative in a degenerate parabolic equation, Visnyk Lviv. Univ. Ser. Mech.-Math. 71 (2009), 78-87. (in Ukrainian)

11. Hryntsiv N.M., Non-local inverse problem for a weakly degenerate parabolic equation, J. of Lviv Polytechnic National University. Physical and Math. Sciences 696 (696), 32-39.

12. Huzyk N., Inverse problem of determining the coefficients in a degenerate parabolic equation, Electron. $J$. Diff. Eq. 2014 (2014), 1-11.

13. Bodnarchuk A., Hryntsiv N., Coefficient inverse problem for a parabolic equation with an arbitrary weak degeneration, Visnyk Lviv. Univ. Ser. Mech.-Math. 75 (2011), 28-42. (in Ukrainian)

14. O. A. Ladyzhenskaya, N. N. Uralceva and V. A. Solonnikov, Linear and quasilinear equations of parabolic type, Moscow, Nauka, 1973. (in Russian) 